\title{
THERMAL STRESSES DUE TO FRICTIONAL HEATING WITH TIME-DEPENDENT SPECIFIC POWER OF FRICTION
}

\author{
Katarzyna TOPCZEWSKA*
}

*Faculty of Mechanical Engineering, Department of Mechanics and Applied Computer Science, Bialystok University of Technology, ul. Wiejska 45C, Bialystok 15-351, Poland

k.topczewska@doktoranci.pb.edu.pl

received 12 July 2017, revised 21 November 2017, accepted 24 November 2017

\begin{abstract}
In this paper influence of temporal profile of the specific friction power (i.e. the product of the coefficient of friction, sliding velocity and contact pressure) on thermal stresses in a friction element during braking was investigated. Spatio-temporal distributions of thermal stresses were analytically determined for a subsurface layer of the friction element, based on the model of thermal bending of a thick plate with unfixed edges (Timoshenko and Goodier , 1970). To conduct calculations, the fields of dimensionless temperature were used. These fields were received in the article (Topczewska, 2017) as solutions to a one-dimensional boundary-value problem of heat conduction for a semi-space heated on its outer surface by fictional heat flux with three, different time profiles of the friction power.
\end{abstract}

Key words: Frictional Heating, Temperature, Thermal Stresses, Time-Dependent Power of Friction

\section{INTRODUCTION}

As a result of friction, on the disc-pad contact surface during braking, high temperature and thermal stresses appear in friction elements. In these conditions the abrasive wear process is intensified and braking efficiency is significantly lower. When thermal stresses exceed the ultimate strength of the friction material, then plastic deformations and thermal cracks on the brake disc surface may arise (Yevtushenko and Kuciej, 2010b; Kim et al. 2013; Wu et al. 2016). Therefore, thermal calculations of working elements are pivotal stage during braking systems design.

Analytical equations to determine temperature and thermal stresses, generated as a result of frictional heating, are predominantly obtain from solution to a one-dimensional thermal problem of friction (Evtushenko et al., 2000; Yevtushenko et al., 2012; 2014). Acceptance of a one-dimensional heat conduction model is reasoned for high values of Peclet number (for braking at high speed) (Blok, 1955). One of the approach to formulate thermal problems of friction during braking is based on a virtual separation of friction elements and heating their working surfaces by the heat fluxes with intensity proportional to the specific power of friction (Talati and Jalalifar, 2009; Yevtushenko and Kuciej, 2010b). Using this approach, in the paper (Evtushenko and Kutsei, 2006) distributions of the non-stationary temperature and thermal stresses for a semi-infinite solid heated on its outer surface by the frictional heat flow modelled by a pulse with rectangular or triangular shape. Thermal problem of friction for a strip - semi-space (pad calliper) tribosystem with linear time profile of frictional heat flux intensity effects on the outer surface of the strip were formulated and solved in the article (Yevtushenko et al., 2011).

In the literature there is a lack of exact solutions to this type of problems with temporal profile of heat flux adequately described the real braking processes. The experimental study (Chichinadze, 1967) shows that this quantity may have a various forms, depends on working conditions, thermal stability of the friction pair and the way of contact pressure regulation.

Influence of temporal profile of specific friction power on spatio-temporal distribution of the temperature in a friction element during single braking was investigated in the article (Topczewska, 2017). For this purpose a one-dimensional boundary-value problem of heat conduction for a homogeneous semi-space (brake disc) heated on its outer surface by the frictional heat flux was formulated and analytically solved. This problem was considered with three different time profiles of specific friction power (Chichinadze, 1967). Obtained solutions determine transient fields of dimensionless temperature in the heated element. Based on received results it was established that temporal profile of the intensity of frictional heat flux has essential influence on temperature distribution in the working elements of brakes.

The main aim of this work is to investigate the influence of selected time profiles of intensity of the frictional heat flux on quasistatic distribution of the thermal stresses in the friction element.

\section{TEMPERATURE}

It was assumed, that a homogeneous half-space (brake disc), from the initial moment $t=t_{0}=0$ to the end of the braking process $\left(t=t_{s}\right)$, is heated on its free surface $z=0$ (friction surface) (Fig. 1) by the frictional heat flux with intensity proportional to the specific power of friction $q_{i}(t)=q_{0} q_{i}^{*}(t), i=1,2,3$ (Chichinadze, 1967):

$q_{1}^{*}(t)=3\left(1-t / t_{s}\right)^{2}, q_{2}^{*}(t)=6 t / t_{s}\left(1-t / t_{s}\right)$,

$q_{3}^{*}(t)=6\left(\sqrt{t / t_{s}}-t / t_{s}\right), \quad 0 \leq t \leq t_{s}$.

where $q_{0}=f V_{0} p_{0}$.

Temporal profiles of the heat flux intensities (1) are characterized by that the braking work in the moment of standstill is equal for all these processes. 


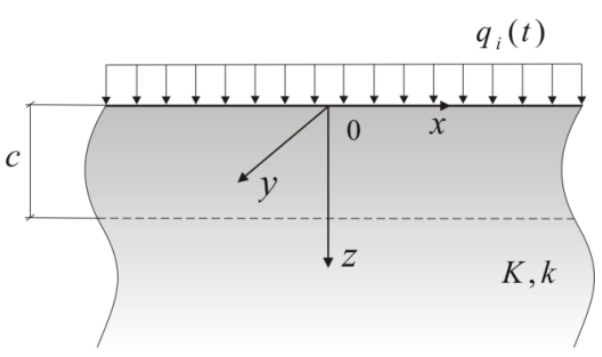

Fig. 1. Scheme of the problem

Solutions to above-mentioned formulated thermal problem of friction, which were found in the paper (Topczewska, 2017), are the following distributions of dimensionless temperature $T_{i}^{*}, i=$ $1,2,3$ :

$T_{1}^{*}(\zeta, \tau)=2 \sqrt{\tau}\left\{3\right.$ ierfc $Z-2 \tau^{*}\left[2\left(1+Z^{2}\right) \operatorname{ierfc} Z-Z \operatorname{Zerfc} Z+\right.$ $\left.0.2 \tau^{* 2}\left[\left(8+18 Z^{2}+4 Z^{4}\right) \operatorname{ierfc} Z-Z\left(7+2 Z^{2}\right) \operatorname{erfc} Z\right]\right\}$,

$\zeta \geq 0,0 \leq \tau \leq \tau_{s}$

$T_{2}^{*}(\zeta, \tau)=4 \tau^{*} \sqrt{\tau}\left\{\left[2\left(1+Z^{2}\right) \operatorname{ierfc} Z-Z \operatorname{Zerfc} Z\right]-0.2 \tau^{*}[(8+\right.$ $\left.\left.\left.18 Z^{2}+4 Z^{4}\right) \operatorname{ierfc} Z-Z\left(7+2 Z^{2}\right) \operatorname{erfc} Z\right]\right\}$,

$\zeta \geq 0,0 \leq \tau \leq \tau_{s}$,

$T_{3}^{*}(\zeta, \tau)=\tau^{*}\left\{3 \sqrt{\pi \tau_{s}}(\operatorname{erfc} Z-2 Z \operatorname{ierfc} Z)-4 \sqrt{\tau}[2(1+\right.$

$\left.\left.\left.Z^{2}\right) \operatorname{ierfc} Z-\operatorname{Zerfc} Z\right]\right\}, \zeta \geq 0,0 \leq \tau \leq \tau_{s}$,

where

$\zeta=\frac{z}{a}, \tau=\frac{k t}{a^{2}}, \tau_{s}=\frac{k t_{s}}{a^{2}}, T_{0}=\frac{q_{0} a}{K}, T_{i}^{*}=\frac{T_{i}-T_{a}}{T_{0}}, Z=\frac{\zeta}{2 \sqrt{\tau}}$,

$a=\sqrt{3 k t_{s}}$ is effective depth of the heat penetration inside brake disc (Chichinadze et al., 1979), $T_{a}$ is initial temperature, $K, k$ are thermal conductivity and thermal diffusivity, respectively.

Based on the solutions (2)-(4) and the model of temperature bending of a thick plate with unfixed edges, the distributions of quasi-static thermal stresses in heated brake disc will be received.

\section{THERMAL STRESSES}

Distribution of the transient temperature field in a brake disc (2)-(4), due to its free surface heating by the frictional heat flux with intensities (1), generate temperature strains and stresses, which may initiate the superficial cracks of the disc material. Experimental research shows that the strongest influence on the material cracking along the direction of the disc sliding, according to the scheme of the process (Fig. 1), has lateral normal component of stress tensor $\sigma_{y}$ (acting along the $y$-axis) (Jewtuszenko et al., 2015). For a one-dimensional model of frictional heating in the direction perpendicular to the friction surface $-z$, lateral and longitudinal normal components of the stresses are equal $\sigma_{y}=\sigma_{x}=\sigma$ (Timoshenko and Goodier, 1970). However, in case of plane state of stresses, normal component of the stress tensor $\sigma_{z}$, which acts in the direction of heating, is negligible.

In accordance with model of thermal bending of a thick strip of the thickness $c=a(\zeta=1)$ with unfixed ends, the dimensionless field of lateral thermal strains $\varepsilon_{y, i}^{*}(\zeta, \tau)=\varepsilon_{i}^{*}(\zeta, \tau)$ we find from the equation (Timoshenko and Goodier, 1970):

$$
\begin{aligned}
\varepsilon_{i}^{*}(\zeta, \tau)=\int_{0}^{1} T_{i}^{*}(\zeta, \tau) d \zeta & \\
& +12(\zeta-0.5) \int_{0}^{1}(\zeta-0.5) T_{i}^{*}(\zeta, \tau) d \zeta
\end{aligned}
$$

$0 \leq \zeta \leq 1,0 \leq \tau \leq \tau_{s}$.

where $T_{i}^{*}(\zeta, \tau), i=1,2,3$ are fields of the dimensionless temperature (2)-(4).

Writing the thermal strains (6) in the form:

$\varepsilon_{i}^{*}(\zeta, \tau)=\bar{T}_{i}^{*}(\tau)(4-6 \zeta)+6 M_{i}^{*}(\tau)(2 \zeta-1)$,

where dimensionless mean temperature $\bar{T}_{i}^{*}(\tau)$ and temperature momentum $M_{i}^{*}(\tau)$, for three intensities of heat flux (1), we determine from the equations:

$\bar{T}_{i}^{*}(\tau)=\int_{0}^{1} T_{i}^{*}(\zeta, \tau) d \zeta, \quad M_{i}^{*}(\tau)=\int_{0}^{1} \zeta T_{i}^{*}(\zeta, \tau) d \zeta$,

and substituting the dimensionless temperatures (2)-(4), to the relations (8), we have:

$\bar{T}_{1}^{*}(\tau)=4 \tau\left\{3 I_{0}(\tau)-2 \tau^{*}\left[2 I_{0}(\tau)+2 I_{2}(\tau)-J_{1}(\tau)\right]+\right.$

$\left.0.2 \tau^{* 2}\left[8 I_{0}(\tau)+18 I_{2}(\tau)+4 I_{4}(\tau)-7 J_{1}(\tau)-2 J_{3}(\tau)\right]\right\}$,

$M_{1}^{*}(\tau)=8 \tau \sqrt{\tau}\left\{3 I_{1}(\tau)-2 \tau^{*}\left[2 I_{1}(\tau)+2 I_{3}(\tau)-J_{2}(\tau)\right]+\right.$

$\left.0.2 \tau^{* 2}\left[8 I_{1}(\tau)+18 I_{3}(\tau)+4 I_{5}(\tau)-7 J_{2}(\tau)-2 J_{4}(\tau)\right]\right\}$

$\bar{T}_{2}^{*}(\tau)=8 \tau \tau^{*}\left\{\left[2 I_{0}(\tau)+2 I_{2}(\tau)-J_{1}(\tau)\right]-0.2 \tau^{*}\left[8 I_{0}(\tau)+\right.\right.$

$\left.\left.18 I_{2}(\tau)+4 I_{4}(\tau)-7 J_{1}(\tau)-2 J_{3}(\tau)\right]\right\}$,

$M_{2}^{*}(\tau)=16 \tau \sqrt{\tau} \tau^{*}\left\{\left[2 I_{1}(\tau)+2 I_{3}(\tau)-J_{2}(\tau)\right]-0.2 \tau^{*}\left[8 I_{1}(\tau)+\right.\right.$

$\left.\left.18 I_{3}(\tau)+4 I_{5}(\tau)-7 J_{2}(\tau)-2 J_{4}(\tau)\right]\right\}$,

$\bar{T}_{3}^{*}(\tau)=2 \sqrt{\tau} \tau^{*}\left\{3 \sqrt{\pi \tau_{s}}\left[J_{0}(\tau)-2 I_{1}(\tau)\right]-8 \sqrt{\tau}\left[I_{0}(\tau)+I_{2}(\tau)-\right.\right.$

$\left.\left.0.5 J_{1}(\tau)\right]\right\}$

$M_{3}^{*}(\tau)=4 \tau \tau^{*}\left\{3 \sqrt{\pi \tau_{s}}\left[J_{1}(\tau)-2 I_{2}(\tau)\right]-8 \sqrt{\tau}\left[I_{1}(\tau)+I_{3}(\tau)-\right.\right.$

$\left.\left.0.5 J_{2}(\tau)\right]\right\}$,

where integrals $I_{k}(\tau)$ and $J_{k}(\tau)$ are defined by the following formulas:

$I_{k}(\tau)=\int_{0}^{X} Z^{k} \operatorname{ierfc} Z d Z, k=0,1,2 \ldots$

$J_{k}(\tau)=\int_{0}^{X} Z^{k} \operatorname{erfc} Z d Z, k=0,1,2 \ldots$

where $Z$ has the form (5) and $X=(2 \sqrt{\tau})^{-1}$.

Integrals $I_{k}(\tau)(15)$, we transform to the following form:

$I_{k}(\tau)=\frac{1}{\sqrt{\pi}} N_{k}(\tau)-J_{k+1}(\tau), k=0,1,2 \ldots$

where $N_{k}(\tau)=\int_{0}^{X} Z^{k} e^{-Z^{2}} d Z, k=0,1,2 \ldots$

Applying the recursive formula (Prudnikov et al., 1998):

$J_{k}(\tau)=\frac{k(k-1)}{2(k+1)} J_{k-2}(\tau)+\left(X^{2}-\frac{k}{2}\right) \frac{X^{k-1}}{k+1} \operatorname{erfc} X-\frac{X^{k} e^{-X^{2}}}{\sqrt{\pi}(k+1)}$,

and $J_{0}(\tau)=\sqrt{\pi}^{-1}-\operatorname{ierfc} X, J_{1}(\tau)=\frac{1}{4} \operatorname{erfc} X-\frac{1}{2} X \operatorname{ierfc} X$,

integrals $J_{k}(\tau), k=0,1,2 \ldots(16)$ we write in the form:

$J_{2}(\tau)=\frac{1}{3 \sqrt{\pi}}\left[1-e^{-X^{2}}\left(X^{2}+1\right)\right]+\frac{X^{3}}{3} \operatorname{erfc} X$,

$J_{3}(\tau)=\frac{1}{8}\left[\frac{3}{2} \operatorname{erf} X-\frac{X e^{-X^{2}}}{\sqrt{\pi}}\left(2 X^{2}+3\right)\right]+\frac{X^{4}}{4} \operatorname{erfc} X$,

$J_{4}(\tau)=\frac{2}{5 \sqrt{\pi}}\left[1-e^{-X^{2}}\left(\frac{1}{2} X^{4}+X^{2}+1\right)\right]+\frac{X^{5}}{5} \operatorname{erfc} X$, 
$J_{5}(\tau)=\frac{5}{8}\left[\frac{1}{2} \operatorname{erf} X-\frac{X e^{-X^{2}}}{3 \sqrt{\pi}}\left(\frac{4}{5} X^{4}+2 X^{2}+3\right)\right]+\frac{X^{6}}{6} \operatorname{erfc} X$,

$J_{6}(\tau)=\frac{6}{7 \sqrt{\pi}}\left[1-e^{-X^{2}}\left(\frac{1}{6} X^{6}+\frac{1}{2} X^{4}+X^{2}+1\right)\right]+\frac{X^{7}}{7} \operatorname{erfc} X$,

Using the relations (Prudnikov et al., 1986):

$N_{k}(\tau)=\frac{k-1}{2} N_{k-2}(\tau)-\frac{X^{k-1}}{2} e^{-X^{2}}, k=0,1,2 \ldots$

where $N_{0}(\tau)=\frac{\sqrt{\pi}}{2}(1-\operatorname{erfc} X), N_{1}(\tau)=\frac{1}{2}\left(1-e^{-X^{2}}\right)$,

we find the integrals $N_{k}(\tau), k=0,1,2 \ldots(18)$ :

$N_{2}(\tau)=\frac{1}{4} \sqrt{\pi} \operatorname{erf} X-\frac{X}{2} e^{-X^{2}}$,

$N_{3}(\tau)=\frac{1}{2}\left[1-e^{-X^{2}}\left(X^{2}+1\right)\right]$,

$N_{4}(\tau)=\frac{3}{8} \sqrt{\pi} \operatorname{erf} X-\frac{X}{2} e^{-X^{2}}\left(X^{2}+\frac{3}{2}\right)$,

$N_{5}(\tau)=1-e^{-X^{2}}\left(\frac{1}{2} X^{4}+X^{2}+1\right)$.

Substituting the relations (20)-(25) and (27)-(31) to the formula (17) we obtain:

$I_{0}(\tau)=\frac{1}{4} \operatorname{erfc} X+\frac{X}{2 \sqrt{\pi}} e^{-X^{2}}-\frac{X^{2}}{2} \operatorname{erfc} X$,

$I_{1}(\tau)=\frac{1}{6 \sqrt{\pi}}\left(1-e^{-X^{2}}\right)+\frac{1}{3} X^{2} \operatorname{ierfc} X$,

$I_{2}(\tau)=\frac{1}{16} \operatorname{erf} X+\frac{X}{8 \sqrt{\pi}} e^{-X^{2}}\left(2 X^{2}-1\right)-\frac{X^{4}}{4} \operatorname{erfc} X$,

$I_{3}(\tau)=\frac{1}{10 \sqrt{\pi}}\left[1+e^{-X^{2}}\left(2 X^{4}-X^{2}-1\right)\right]-\frac{X^{5}}{5} \operatorname{erfc} X$,

$I_{4}(\tau)=\frac{1}{16} \operatorname{erf} X+\frac{X}{24 \sqrt{\pi}} e^{-X^{2}}\left(4 X^{4}-2 X^{2}-3\right)-\frac{X^{6}}{6} \operatorname{erfc} X$,

$I_{5}(\tau)=\frac{1}{7 \sqrt{\pi}}\left[1+e^{-X^{2}}\left(X^{6}-\frac{1}{2} X^{4}-X^{2}-1\right)\right]-\frac{X^{7}}{7} \operatorname{erfc} X$.

Substituting the solutions (20)-(25) and (32)-(37) to the dimensionless mean temperature $\bar{T}_{i}^{*}(\tau)$ and temperature momentum $M_{i}^{*}(\tau)(9)-(14)$, and received results to the equation (7), we obtain fields of dimensionless lateral deformations $\varepsilon_{\mathrm{i}}^{*}(\zeta, \tau), \mathrm{i}=$ $1,2,3$.

Knowing the dimensionless temperature (2)-(4) and thermal strains (7), distributions of the dimensionless thermal stresses $\sigma_{i}^{*}(\zeta, \tau)$ in the brake disc for three intensities of the frictional heat flux (1), we determine from the equations (Timoshenko and Goodier, 1970):

$\sigma_{i}^{*}(\zeta, \tau)=\varepsilon_{i}^{*}(\zeta, \tau)-T_{i}^{*}(\zeta, \tau)$,

$0 \leq \zeta \leq 1,0 \leq \tau \leq \tau_{s}, i=1,2,3$

where $\sigma_{i}(\zeta, \tau)=\sigma_{0} \sigma_{i}^{*}(\zeta, \tau), \sigma_{0}=\frac{\alpha E T_{0}}{1-v}$.

$\mathrm{E}$ is Young's modulus, $\alpha$ is linear thermal expansion coefficient and $v$ is Poisson's ratio.

\section{NUMERICAL ANALYSIS}

Based on obtained analytical solutions, numerical analysis of the transient distributions of the temperature and quasi-static thermal stresses in a brake disc (half-space) for selected braking modes, i.e. for three different intensities of frictional heat fluxes (1). The following dimensionless parameters: spatial coordinate $\zeta$, time $\tau$ and braking time $\tau_{S}=1$, which are determined by the equations (5) were used to perform numerical calculations.
Spatio-temporal distributions of the dimensionless temperature $T_{i}^{*}(\zeta, \tau), i=1,2,3$ generated due to frictional heating of the working surface $\zeta=0$ by the heat fluxes with three intensities (1) and corresponding distributions of the quasi-static normal thermal stresses $\sigma_{i}^{*}(\zeta, \tau), i=1,2,3$ were presented in the Fig. 2. As follows from the functions (equations (1)), which describe the intensities of the frictional heat fluxes effects on the working surface of the brake disc, the profile of the first one $q_{1}^{*}(\tau)$ is characterized by the fact, that the maximum value occurs at the initial moment of braking $(\tau=0)$ and afterwards it decreases with time to zero at the moment of standstill $\tau=\tau_{S}=1$. However intensities $q_{i}^{*}(\tau), i=2,3$ increase from zero at the initial moment $(\tau=0)$ to the maximum values at $0.5 \tau_{s}$ for $i=2$ and at $0.25 \tau_{s}$ for $i=3$. After reached maximum value of the heat flux intensities they decrease to zero at the stop moment. Distributions of the temperature inside the brake disc, generated due to heating friction surface by the heat flux with the foregoing intensities, are shown in Fig. 2 a,c,e. Conducted analysis of the spatio-temporal distributions of temperature allow to establish, that the time of appearance of the maximum heat flux value has crucial influence both on the time of appearance maximum temperature on the friction surface, and on the temperature distribution inside the brake disc. If the maximum value of heat flux occurs in the initial stage of braking $\left(q_{1}^{*}(\tau)\right)$ maximum value of temperature $T_{1 \text {,max }}^{*}=$ 1.2 is reached at $\tau_{\max } \cong 0.317 \tau_{s}$. The time of appearance of the maximum temperature on the working surface of the disc increases with increasing the time to reach maximum value of frictional heat flux. For the intensities of heat flux $q_{2}^{*}(\tau)$ time $\tau_{\text {max }}=0.75 \tau_{s}\left(T_{2, \text { max }}^{*}=1.17\right)$ and for $q_{3}^{*}(\tau) \tau_{\max } \cong 0.62 \tau_{s}$ $\left(T_{3, \max }^{*}=1.09\right)$.

In Fig. $2 b, d, f$, the dimensionless distributions of the quasistatic thermal stresses $\sigma_{i}^{*}, i=1,2,3$ are presented, corresponding to the spatial-time distributions of the dimensionless temperature (Fig. 2a,c,e.) in the brake disc. Overall character of the change of stresses state in the consider zone of the brake disc $0 \leq \zeta \leq 1$ is similar for all analyzed time profiles of the frictional heat flux intensities. In the initial stage of braking, in the subsurface region of the disc $0 \leq \zeta \leq 0.2$, the compressive stresses $\sigma_{i}^{*}<0$ are generated. Value of these stresses decreases with time, to the sign change - which means that the tensile stresses take place. The time of change compressive stresses $\sigma_{i}^{*}<0$ into tensile stresses $\sigma_{i}^{*}>0$ depends on the time of appearance of maximum temperature value on the friction surface of the disc, i.e. the faster the maximum temperature is reached, the earlier becomes that change. In the case of heat flux intensity $q_{1}^{*}(\tau)$ when the temperature achieved the greatest value at the time moment $\tau_{\max } \cong 0.317 \tau_{s}$ the time of the change of stress sign (appearance of the isoline of zero stress level) on the working surface and inside the disc is equal to $\tau=0.53$ (Fig. 2b). Whereas for the fluxes $q_{i}^{*}(\tau), i=2,3$ the change of the compressive stresses into tensile stresses, in the consider zone $0 \leq \zeta \leq 0.2$, occurs at the time $\tau=0.88$ (Fig. 2d) and $\tau=0.8$ (Fig. 2f). Similar character of the stresses appearance and its change can be observed in the region $0.75 \leq \zeta \leq 1$. In the early stage of the heating process, the compressive stresses are generated, but their values are lower in comparison with the subsurface zone of the disc. Afterwards the stresses values decreases with time, to the sign change and the tensile stresses appear, in the similar way as in the subsurface region but also with lower values. 

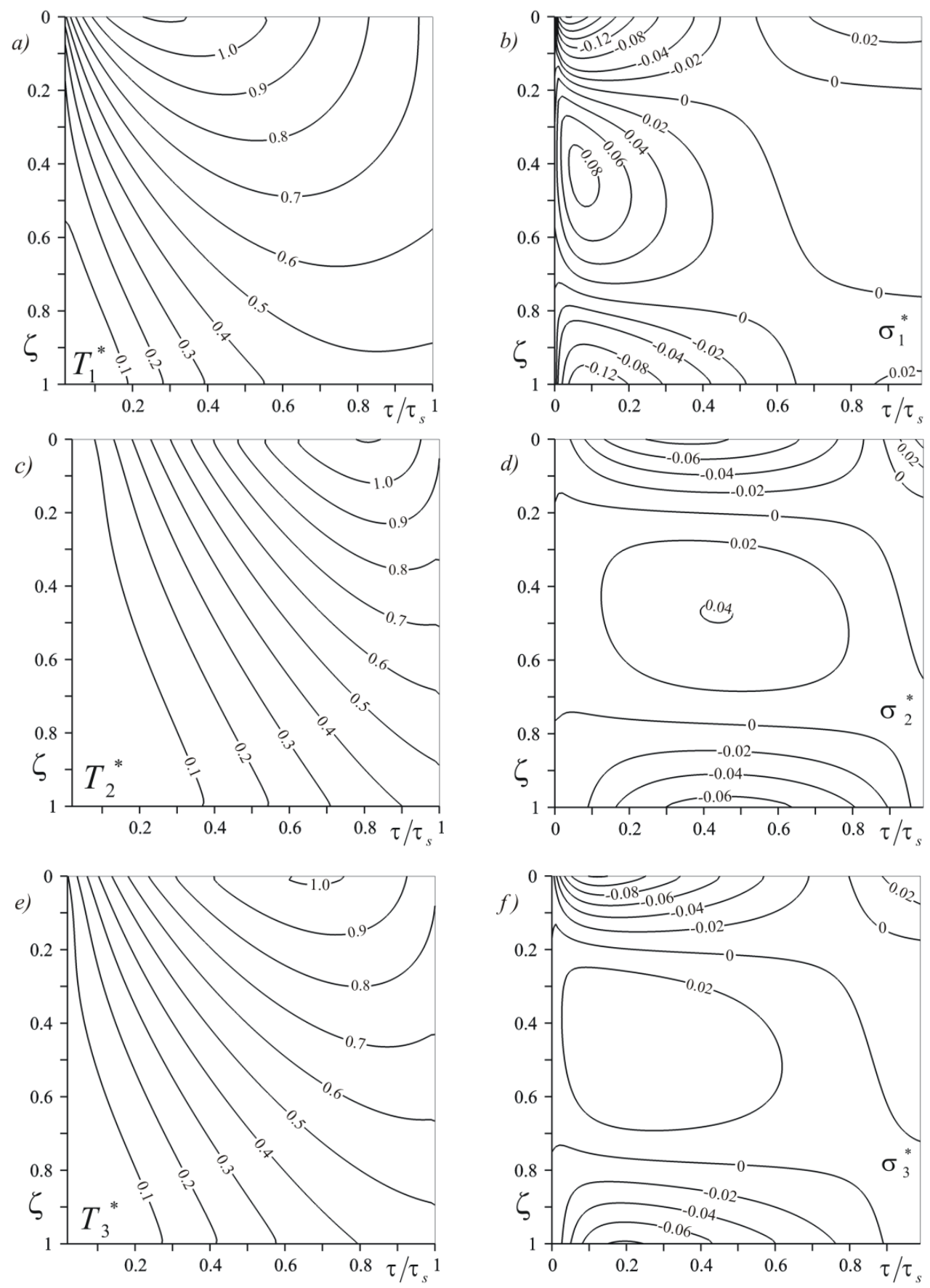

Fig. 2. Spatio-temporal distributions of the dimensionless temperatures $\left.\left.\left.T_{i}^{*}: a\right) i=1 ; c\right) i=2 ; e\right) i=3$; and the transverse thermal stresses $\left.\left.\left.\sigma_{i}^{*}: b\right) i=1 ; d\right) i=2 ; f\right) i=3$; inside the brake disc, for three time profiles of the heat flux intensities

Between the above-mentioned regions, in the initial stage of braking, the tensile stresses are generated, which in the all consider cases of frictional heating is present in the whole time interval $0<\tau \leq \tau_{s}$. The greatest value of the normal compressive stresses in this region occurs in the case of the frictional heating of the disc by the heat flux with intensity $q_{1}^{*}(\tau)$ and is equal $\sigma_{1}^{*}=0.08$ (Fig. 2b). Stresses appear in this zone, in the early stage of braking - at time moment $\tau \approx 0.04$. The time of occurrence of the maximum compressive stresses is almost equal to the time of appearance of the maximum values of tensile stresses on the heated surface, and for intensities of frictional heat fluxes $q_{i}^{*}(\tau), i=2,3$ is $\tau \approx 0.4$ (Fig. $2 \mathrm{~d}$ ) and $\tau \approx 0.16$ (Fig. 2f), respectively. After reaching the maximum value, these stress decrease with time, and the region of its occurrence "narrows" and "moves" towards the depth $\zeta=1$, to the replacement of the compressive stresses, adjacent to this region.

Evolutions of the dimensionless thermal stresses on the friction surface of the disc for three frictional heating modes are presented in Fig. 3. On the friction surface $\zeta=0$, the maximum values of the dimensionless compressive stresses $\sigma_{1, \text { min }}^{*}=$ $-0.29, \sigma_{2, \min }^{*}=-0.12, \sigma_{3, \min }^{*}=-0.13$ are reached at the time moments $\tau=0.04, \tau=0.4$ and $\tau=0.16$, respectively. After achieving the greatest value of the temperature (Fig. 2 a,c,e), compressive stresses on the heated surface disappear with time, and at the moments $\tau=0.53\left(q_{1}^{*}(\tau)\right), \tau=0.88 \quad\left(q_{2}^{*}(\tau)\right)$ and $\tau=0.8\left(q_{3}^{*}(\tau)\right)$ the sign of thermal stress change and tensile stresses appear, accompanied by the temperature decrease to the standstill moment. In cases $q_{i}^{*}(\tau), i=2,3$ tensile stresses 
monotonically increase and reach maximum values $\sigma_{2, \max }^{*}=$ 0.05 and $\sigma_{3, \max }^{*}=0.04$ at the end of the braking process $\tau=\tau_{S}=1$. Whereas, in the process $q_{1}^{*}(\tau)$ stresses increase, reaching the maximum value $\sigma_{1, \max }^{*}=0.04$ at the moment $\tau=0.88$ and next, we can observe a slight fall of this value to the standstill.

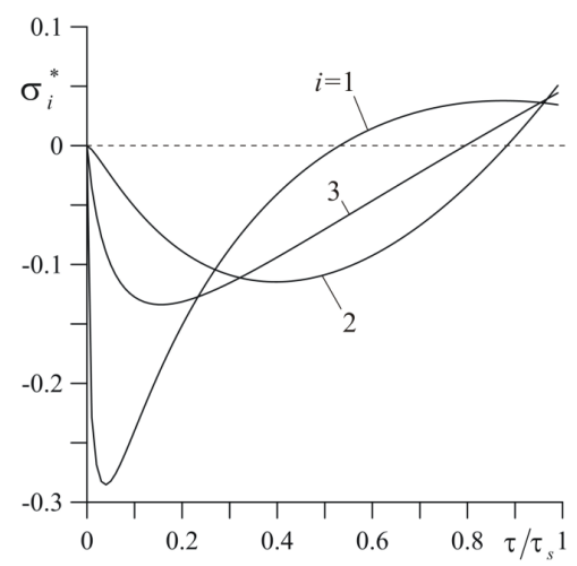

Fig. 3. Evolutions of the dimensionless transverse thermal stresses $\sigma_{i}^{*}, i=1,2,3$ for three temporal profiles of the intensities of frictional heat flux, on the working surface of the disc $\zeta=0$.

\section{CONCLUSIONS}

In this paper fields of the dimensionless thermal stresses in the brake disc heated by the frictional heat flux with intensity defined by three different temporal profiles were analytically determined. Conducted analysis allow us to make the following conclusions:

- in the initial stage of braking, adjacent to the friction surface the compressive stresses appear, subsequently decrease to zero, then the tensile stress zone arise and persist until the end of braking;

- increase of the time of achieving the maximum value of the specific friction power, results in increase of the time of occurrence of stress sign change on the heated surface;

- rapid decrease of the disc temperature before the stop moment, causes the appearance of the tensile stresses with higher value on the friction surface. Exceed of the ultimate strength of the friction material by the value of this stress can cause the initiation of the superficial thermal cracks;

- values and distribution of the thermal stresses, generated inside the heated brake disc, depends mainly on temporal profile of the specific friction power.

Nomenclature: $a$ - effective depth of heat penetration [m]; $c$ - thickness of the strip [m]; $E$ - Young's modulus [MPa]; erf(x) - Gauss error function; $\operatorname{erfc}(\mathrm{x})=1-\operatorname{erf}(\mathrm{x})-\operatorname{complementary}$ error function; ierfc $(\mathrm{x})=$ $\pi^{-1 / 2} \exp \left(-\mathrm{x}^{2}\right)-\operatorname{xerfc}(\mathrm{x})-$ integral of complementary error function; $K$ - thermal conductivity [W K $\left.{ }^{-1} \mathrm{~m}^{-1}\right] ; k-$ thermal diffusivity $\left[\mathrm{m}^{2} \mathrm{~s}^{-1}\right.$; $q$ - intensity of the heat flux [W $\left.\mathrm{m}^{-2}\right] ; q^{*}-$ dimensionless intensity of the heat flux; $T$ - temperature $[\mathrm{K}] ; T^{*}$ - dimensionless temperature; $T_{a}$ - initial temperature [K]; $T_{0}$-temperature scaling factor $[\mathrm{K}] ; t$ - time $[\mathrm{s}] ; t_{s}-$ braking time [s]; $x y z$ - spatial coordinates [m]; $\alpha$ - linear thermal expansion coefficient $\left[\mathrm{K}^{-1}\right] ; \varepsilon^{*}-$ dimensionless thermal strain; $\sigma_{0}$-stress scaling factor [MPa]; $\sigma$ - normal component of stress tensor [MPa]; $\sigma^{*}$ dimensionless component of stress tensor; $\tau$ - dimensionless time (Fourier number); $\tau_{s}$-dimensionless braking time; $\zeta$-dimensionless depth.

\section{REFERENCES}

1. Abramowitz M., Stegun I.A. (1972), Handbook of Mathematical Functions with Formulas, Graphs, and Tables, National Bureau of Standards, Washington.

2. Blok H. (1955) The dissipation of frictional heat, Applied Scientific Research, Section A. (2-3), 151-181.

3. Chichinadze A.V. (1967) Calculation and study of external friction during braking, Nauka, Moscow, (in Russian).

4. Chichinadze A.V., Braun E.D., Ginsburg A.G., Ignat'eva Z.V. (1979), Calculation, Test and Selection of Frictional Couples, Nauka, Moscow (in Russian).

5. Evtushenko A., Kutsei M. (2006) Initiating of thermal cracking of materials by frictional heating, Journal of Friction and Wear, 27(2), 9-16.

6. Evtushenko 0.0., Ivanyk E.H., Horbachova N.V. (2000) Analytic methods for thermal calculation of brakes, Materials Science, 36(6), 857-862.

7. Jewtuszenko O., Kuciej M., Tołstoj-Sienkiewicz J. (2015) Transients and quasi-stationary friction temperature fields, Oficyna Wydawnicza Politechniki Białostockiej, Białystok (in Polish).

8. Kim S.W., Segu D.Z., Kim S.S. (2013) The Thermo-mechanical Cracking Analysis of Break System, Procedia Engineering, 68, 586-592.

9. Prudnikov A.P., Brychkov Yu. A., Marichev O. I. (1986), Integrals and Series, Vol. 1: Elementary Functions, Gordon and Breach, New York.

10. Prudnikov A. P., Brychkov Yu. A., Marichev O. I. (1998) Integrals and Series, Vol. 2, Special Functions, New York-London,Taylor \& Francis.

11. Talati F., Jalalifar S. (2009) Analysis of heat conduction in a disk brake system, Heat Mass Transfer, 45, 1047-1059.

12. Timoshenko S.P., Goodier J.N. (1970) Theory of Elasticity, McGraw-Hill, New York.

13. Topczewska K. (2017) Frictional heating with time-dependent specific power of friction, Acta Mechanica et Automatica, 11(2), 111-115.

14. Wu S.C., Zhang S.Q., Xu Z.W. (2016) Thermal crack growth-based fatigue life prediction due to braking for a high-speed railway brake disc, International Journal of Fatigue, 87, 359-369.

15. Yevtushenko A.A., Kuciej M. (2010a) Two heat conduction problems with frictional heating during braking, J. Theor. Appl. Mech., 48(2), 367-380.

16. Yevtushenko A.A., Kuciej M. (2010b) Two calculation schemes for determination of thermal stresses due to frictional heating during braking, Journal of Theoretical and Applied Mechanics, 48(3), 605-621.

17. Yevtushenko A.A., Kuciej M., Yevtushenko O.O. (2011) Temperature and thermal stresses in material of a pad during braking, Arch. Appl. Mech., 81, 715-726.

18. Yevtushenko A.A., Kuciej M. (2012) One-dimensional thermal problem of friction during braking: The history of development and actual state, International Journal of Heat and Mass Transfer, 55(15), 4148-4153.

19. Yevtushenko A.A., Kuciej M., Yevtushenko O. (2014), The asymptotic solutions of heat problem of friction for a three-element tribosystem with generalized boundary conditions on the surface of sliding, International Journal of Heat and Mass Transfer, 70, 128-136.

This work is part of the project no. MB/WM/18/2017 which is carried out in the Faculty of Mechanical Engineering, Bialystok University of Technology. 